\title{
THE WEAK BOUNDED APPROXIMATION PROPERTY OF PAIRS
}

\author{
DONGYANG CHEN, JU MYUNG KIM, AND BENTUO ZHENG \\ (Communicated by Thomas Schlumprecht)
}

Abstract. We study the weak bounded approximation property (weak BAP) of pairs and show that each of the spaces $c_{0}$ and $\ell_{1}$ has a subspace having the approximation property but failing the weak BAP.

\section{INTRODUCTION}

A Banach space $X$ is said to have the approximation property (AP) if $i d_{X} \in$ $\overline{\mathcal{F}}(X)^{\tau_{c}}$, where $i d_{X}$ is the identity map on $X, \mathcal{F}(X)$ is the space of finite rank operators on $X$ and $\tau_{c}$ is the topology of uniform convergence on each compact subset of $X$. If $i d_{X} \in \overline{\{S \in \mathcal{F}(X):\|S\| \leq \lambda\}}{ }^{\tau_{c}}$ for some $\lambda \geq 1$, then we say that $X$ has the $\lambda$-bounded approximation property $(\lambda$-BAP). If $\lambda=1, X$ is said to have the metric approximation property (MAP). Grothendieck 5 ] systematically investigated the AP and the BAP in various points of view. One of the main goals of Banach space theory is to discover the relationship between the AP and the BAP. It was shown that the AP and the BAP are equivalent to the MAP for separable dual spaces [5] (cf. [13, Theorem 1.e.15]). But Figiel and Johnson [3] showed that the AP does not imply the BAP in general. To be more precise, they constructed a Banach space with separable dual which has the AP but fails the BAP. Recently, Figiel, Johnson and Pelczyński [4, Corollary 1.13] introduced the notion of BAP for pairs and showed that each of the spaces $c_{0}$ and $\ell_{1}$ has a subspace having the AP but failing the BAP.

In [12] the notion of weak bounded approximation property (weak BAP) was introduced by Lima and Oja, in an attempt to cope with the old open problem whether for a dual Banach space the AP implies the MAP. For $\lambda \geq 1$, a Banach space $X$ is said to have the weak $\lambda$-BAP if for every Banach space $Z$ and every

Received by the editors January 7, 2013 and, in revised form, August 13, 2013 and September $12,2013$.

2010 Mathematics Subject Classification. Primary 46B28; Secondary 47L20.

Key words and phrases. Approximation property, bounded approximation property, weak bounded approximation property.

The first author was supported in part by the National Natural Science Foundation of China (Grant No. 10701063) and the Fundamental Research Funds for the Central Universities (Grant No. 2011121036).

The second author, who was the corresponding author, was supported by NRF Grant 2013R1A1A2A10058087, funded by the Korean Government.

The third author was partially supported by NSF-DMS-1200370.

The authors were participants in the NSF Workshop in Analysis and Probability, Texas A\&M University (2012). 
$R \in \mathcal{W}(X, Z)$, the space of all weakly compact operators from $X$ into $Z$, we have

$$
i d_{X} \in \overline{\{S \in \mathcal{F}(X):\|R S\| \leq \lambda\|R\|\}}{ }^{\tau_{c}} .
$$

In [12, Proposition 2.2 and Theorem 3.6], it was shown that the AP is strictly weaker than the weak BAP, but they are both equivalent to the weak MAP for dual spaces. The BAP implies the weak BAP, but it is not known whether the converse holds. Lima and Oja [12] conjectured that they are different properties. The main result of this paper is the following theorem.

Theorem 1.1. If $X$ has the AP but $X^{*}$ fails to have the AP, then there exists a subspace $Y$ of $c_{0}(X)$ (respectively, $\ell_{p}(X)(1 \leq p<\infty)$ ) such that $Y$ has the AP but fails the weak BAP.

Here $c_{0}(X)$ (resp. $\ell_{p}(X)$ ) is the Banach space of all null sequences (resp. absolutely $p$-summable sequences) in $X$. The proof of Theorem 1.1 uses the techniques in 4 and some characterizations of the weak BAP of pairs. We therefore prove Theorem 1.1 in Section 3 after we obtain the results about the weak BAP of pairs in Section 2.

Corollary 1.2. Each of the spaces $c_{0}$ and $\ell_{1}$ has a subspace which has the AP but fails the weak BAP.

Proof. The case $c_{0}$ : There exists a subspace $X$ of $c_{0}$ which has a basis so that $X^{*}$ fails the AP [7, Corollary JS]. It follows from Theorem 1.1 that there exists a subspace $Y$ of $c_{0}(X) \subset c_{0}$ such that $Y$ has the AP but fails the weak BAP.

The case $\ell_{1}$ : Let $X$ be a subspace of $\ell_{1}$ which fails the AP [15]. Let $\left(G_{n}\right)_{n}$ be a sequence of finite-dimensional subspaces of $X$ which is dense in the class of all finite-dimensional subspaces of $X$. Let $C_{1}=\left(\sum_{n} G_{n}\right)_{\ell_{1}}$. Then by [6, Theorem 3] $C_{1}^{*}$ fails the AP. Obviously, $C_{1}$ has the AP. It follows from Theorem 1.1 that there exists a subspace $Y$ of $\ell_{1}\left(C_{1}\right) \subset \ell_{1}$ such that $Y$ has the AP but fails the weak BAP.

Remark 1.3. The case $c_{0}$ in Corollary 1.2 can also be deduced from 4, Corollary 1.13] because the weak $\lambda$-BAP and the $\lambda$-BAP are equivalent for Banach spaces with separable dual (see [14, Corollary 1]).

\section{The BOUnded APPROXIMATION PROPERTY OF PAIRS}

Figiel, Johnson and Pelczyński [4] introduced and investigated the BAP of pairs. Let $Y$ be a closed subspace of a Banach space $X$. Let $\lambda \geq 1$. The pair $(X, Y)$ is said to have the $\lambda$-BAP if for every finite-dimensional subspace $F$ of $X$ and for every $\varepsilon>0$ there exists an $S \in \mathcal{F}(X)$ with $\|S\| \leq \lambda+\varepsilon$ such that $S x=x$ for all $x \in F$ and $S(Y) \subset Y$. The pair $(X, X)$ has the $\lambda$-BAP if and only if $X$ has the $\lambda$ BAP (see [8, Proposition 1.1]). We proceed in a more general setting in this paper to include other approximation properties. We say that the pair $(X, Y)$ has the $\lambda$-A-BAP if the space $\mathcal{F}(X)$ is replaced by a linear subspace $\mathcal{A}(X)$ of $\mathcal{L}(X)$. In [2], the $\lambda$-K -BAP of pairs was studied, where $\mathcal{K}$ is the space of all compact operators between Banach spaces.

The pair $(X, Y)$ is said to have the weak $\lambda$-A-BAP if for every Banach space $Z$ and every $R \in \mathcal{W}(X, Z)$, for every finite-dimensional subspace $F$ of $X$ and for every $\varepsilon>0$, there exists an $S \in \mathcal{A}(X)$ with $\|R S\| \leq(\lambda+\varepsilon)\|R\|$ such that $S x=x$ for all $x \in F$ and $S(Y) \subset Y$. The pair $(X, Y)$ is said to have the weak $\lambda$-BAP if 
$\mathcal{A}=\mathcal{F}$. In this section, we give some characterizations of the weak BAP of pairs. The following lemma [9, Proposition 3.1] is needed in our proof.

Lemma 2.1. Let $Y$ be a closed subspace of $X$. Let $T \in \mathcal{L}(X)$ and let $\mathcal{A}(X)$ be a convex subset of $\mathcal{L}(X)$ and $\alpha>0$. Then the following statements are equivalent:

(a) For every reflexive Banach space $Z$ and every $R \in \mathcal{W}(X, Z)$, we have

$$
R T \in \overline{\{R S: S \in \mathcal{A}(X),\|R S\| \leq \alpha\|R\|, S(Y) \subset Y\}}{ }^{\tau_{c}} .
$$

(b) For every Banach space $Z$ and every $R \in \mathcal{W}(X, Z)$, we have

$$
T \in \overline{\{S \in \mathcal{A}(X):\|R S\| \leq \alpha\|R\|, S(Y) \subset Y\}^{\tau_{c}}} \text {. }
$$

Proposition 2.2. Let $Y$ be a closed subspace of $X$ and let $\lambda \geq 1$. Suppose that $\mathcal{A}(X)$ is a linear subspace of $\mathcal{L}(X)$, which contains $\mathcal{F}(X)$. Then the following statements are equivalent:

(a) For every Banach space $Z$ and every $R \in \mathcal{W}(X, Z)$, for every $\delta>0$ we have

$$
R \in \overline{\{R S: S \in \mathcal{A}(X),\|R S\| \leq(\lambda+\delta)\|R\|, S(Y) \subset Y\}^{\tau_{c}}} .
$$

(b) For every Banach space $Z$ and every $R \in \mathcal{W}(X, Z)$, we have

$$
i d_{X} \in \overline{\{S \in \mathcal{A}(X):\|R S\| \leq \lambda\|R\|, S(Y) \subset Y\}^{\tau_{c}}} .
$$

(c) For every Banach space $Z$ and every $R \in \mathcal{W}(X, Z)$, for every finite-dimensional subspace $F$ of $X$ and for every $\varepsilon>0$, there exists an $S \in \mathcal{A}(X)$ with $\|R S\| \leq \lambda\|R\|$ such that $\|S x-x\| \leq \varepsilon\|x\|$ for all $x \in F$ and $S(Y) \subset Y$.

(d) The pair $(X, Y)$ has the weak $\lambda-\mathcal{A}-B A P$.

Proof. $(\mathrm{b}) \Rightarrow(\mathrm{c})$ is clear.

(c) $\Rightarrow$ (d) This proof is essentially due to the proof of [4, Lemma 1.5]. Let $Z$ be a Banach space and let $R \in \mathcal{W}(X, Z)$. Let $F$ be a finite-dimensional subspace of $X$ and let $\varepsilon>0$. Then we can find a projection $P$ from $X$ onto $F$ with $P(Y) \subset$ $Y$. Choose $\delta>0$ such that $\delta\|P\|<\varepsilon$. By (c) there exists an $S \in \mathcal{A}(X)$ with $\|R S\| \leq \lambda\|R\|$ such that $\|S x-x\| \leq \delta\|x\|$ for all $x \in F$ and $S(Y) \subset Y$. Put $S_{0}=S+\left(i d_{X}-S\right) P \in \mathcal{A}(X)$. Then we have that $S_{0} x=x$ for all $x \in F$, $S_{0}(Y) \subset Y$, and

$$
\begin{aligned}
\left\|R S_{0}\right\| & =\left\|R S+R\left(i d_{X}-S\right) P\right\| \\
& \leq\|R S\|+\|R\|\left\|\left(i d_{X}-S\right) P\right\| \leq \lambda\|R\|+\|R\| \delta\|P\| \leq(\lambda+\varepsilon)\|R\| .
\end{aligned}
$$

Hence the pair $(X, Y)$ has the weak $\lambda$ - $\mathcal{A}$-BAP.

(d) $\Rightarrow$ (a) Let $Z$ be a Banach space and let $R \in \mathcal{W}(X, Z)$. Let $\delta>0$. Let $K$ be a compact subset of $X$ and let $\varepsilon>0$. Choose a $\gamma>0$ so that

$$
(\lambda+\delta+1)\|R\| \gamma \leq \varepsilon
$$

Let $\left\{x_{i}\right\}_{i=1}^{n} \subset K$ be a $\gamma$-net for $K$. Then by (d) there exists an $S \in \mathcal{A}(X)$ with $\|R S\| \leq(\lambda+\delta)\|R\|$ such that $S x_{i}=x_{i}$ for all $1 \leq i \leq n$ and $S(Y) \subset Y$.

Now let $x \in K$. Then there exists an $x_{i_{0}}$ for some $1 \leq i_{0} \leq n$ such that $\left\|x-x_{i_{0}}\right\| \leq \gamma$. We have that

$$
\begin{aligned}
\|R x-R S x\| & \leq\left\|R x-R x_{i_{0}}\right\|+\left\|R x_{i_{0}}-R S x_{i_{0}}\right\|+\left\|R S x_{i_{0}}-R S x\right\| \\
& \leq \gamma\|R\|+(\lambda+\delta) \gamma\|R\| \leq \varepsilon .
\end{aligned}
$$

Hence we obtain assertion (a). 
(a) $\Rightarrow$ (b) Let $Z$ be a Banach space and let $R \in \mathcal{W}(X, Z)$. Let $K$ be a compact subset of $X$ and let $\varepsilon>0$. Choose a $\delta>0$ so that $(\delta /(\lambda+\delta)) \sup _{x \in K}\|R x\| \leq \varepsilon / 2$. By (a) there exists an $S \in \mathcal{A}(X)$ with $\|R S\| \leq(\lambda+\delta)\|R\|$ such that

$$
\sup _{x \in K}\|R S x-R x\| \leq \varepsilon / 2
$$

and $S(Y) \subset Y$. Put $S_{0}=(\lambda /(\lambda+\delta)) S \in \mathcal{A}(X)$. Then we have that $S_{0}(Y) \subset Y$, $\left\|R S_{0}\right\| \leq \lambda\|R\|$, and

$$
\sup _{x \in K}\left\|R S_{0} x-R x\right\| \leq \frac{\lambda}{\lambda+\delta} \sup _{x \in K}\|R S x-R x\|+\frac{\delta}{\lambda+\delta} \sup _{x \in K}\|R x\| \leq \varepsilon .
$$

Hence $R \in \overline{\{R S: S \in \mathcal{A}(X),\|R S\| \leq \lambda\|R\|, S(Y) \subset Y\}}^{\tau_{c}}$. By Lemma 2.1 we obtain assertion (b).

We can also extend [4, Lemma 1.5] as the following proposition.

Proposition 2.3. Let $Y$ be a closed subspace of $X$ and let $\lambda \geq 1$. Suppose that $\mathcal{A}(X)$ is a linear subspace of $\mathcal{L}(X)$, which contains $\mathcal{F}(X)$. Then the following statements are equivalent:

(a) $i d_{X} \in \overline{\{S \in \mathcal{A}(X):\|S\| \leq \lambda, S(Y) \subset Y\}}^{\tau_{c}}$.

(b) For every finite-dimensional subspace $F$ of $X$ and for every $\varepsilon>0$, there exists an $S \in \mathcal{A}(X)$ with $\|S\| \leq \lambda$ such that $\|S x-x\| \leq \varepsilon\|x\|$ for all $x \in F$ and $S(Y) \subset Y$.

(c) The pair $(X, Y)$ has the $\lambda-\mathcal{A}-B A P$.

Proof. $(\mathrm{a}) \Rightarrow(\mathrm{b})$ is clear and we use the proof of [4, Lemma 1.5] to show $(\mathrm{b}) \Rightarrow(\mathrm{c})$. As in the proof of Proposition 2.2 we can use a simple perturbation argument to show $(\mathrm{c}) \Rightarrow(\mathrm{a})$.

The following theorem is the main result in this section. Here $\mathcal{A}^{*}(X):=\left\{S^{*}\right.$ : $S \in \mathcal{A}(X)\}$.

Theorem 2.4. Suppose that $\mathcal{A}(X)$ is a linear subspace of $\mathcal{K}(X)$, which contains $\mathcal{F}(X)$. Then the following statements are equivalent:

(a) $i d_{X^{*}} \in{\overline{\mathcal{A}^{*}(X)}}^{\tau_{c}}$.

(b) For every finite-codimensional subspace $Y$ of $X$, the pair $(X, Y)$ has the weak $\mathcal{A}-M A P$.

(c) There exists a $\lambda \geq 1$ satisfying the fact that for every finite-codimensional subspace $W$ of $X$, there exists a finite-codimensional subspace $Y$ of $X$ with $Y \subset W$ such that the pair $(X, Y)$ has the weak $\lambda-\mathcal{A}$-BAP.

Since $\mathcal{F}\left(X^{*}\right) \subset{\overline{\mathcal{F}^{*}(X)}}^{\tau_{c}}$ (see [13, Lemma 1.e.17]), we have:

Corollary 2.5. The following statements are equivalent:

(a) $X^{*}$ has the AP.

(b) For every finite-codimensional subspace $Y$ of $X$, the pair $(X, Y)$ has the weak MAP.

(c) There exists a $\lambda \geq 1$ satisfying the fact that for every finite-codimensional subspace $W$ of $X$, there exists a finite-codimensional subspace $Y$ of $X$ with $Y \subset W$ such that the pair $(X, Y)$ has the weak $\lambda$-BAP. 
Theorem $2.4(\mathrm{~b}) \Rightarrow(\mathrm{c})$ is clear, and we use the argument in the proof [4, Proposition 1.6] to show the other parts. We need the following result to show Theorem $2.4(\mathrm{a}) \Rightarrow(\mathrm{b})$.

Proposition 2.6. Suppose that $\mathcal{A}(X)$ is a convex subset of $\mathcal{K}(X)$ with $0 \in \mathcal{A}(X)$ and let $T \in \mathcal{L}(X)$. If $T^{*} \in{\overline{\mathcal{A}^{*}(X)}}^{\tau_{c}}$, then for every Banach space $Z$ and every $R \in \mathcal{W}(X, Z)$ there exists a net $\left(T_{\alpha}\right)$ in $\mathcal{A}(X)$ with $\left\|R T_{\alpha}\right\| \leq\|T\|\|R\|$ for all $\alpha$ such that

$$
T_{\alpha} \stackrel{\tau_{c}}{\longrightarrow} T \text { and } T_{\alpha}^{*} \stackrel{\tau_{c}}{\longrightarrow} T^{*} .
$$

In order to show Proposition 2.6, we need the following lemma which is contained in [10, Theorem 5.4].

Lemma 2.7. Suppose that $\mathcal{A}(X)$ is a convex subset of $\mathcal{K}(X)$ with $0 \in \mathcal{A}(X)$ and let $T \in \mathcal{L}(X)$. If $T \in \overline{\mathcal{A}(X)}^{\tau_{c}}$, then for every Banach space $Z$ and every $R \in \mathcal{W}(Z, X)$, we have

$$
T \in \overline{\{S \in \mathcal{A}(X):\|S R\| \leq\|T\|\|R\|\}}^{\tau_{c}} .
$$

Proof of Proposition 2.6. Let $Z$ be a Banach space and let $R \in \mathcal{W}(X, Z)$. Since $T^{*} \in \overline{\mathcal{A}}^{*}(X){ }^{\tau_{c}}$, by Lemma 2.7 there exists a net $\left(S_{\beta}\right)$ in $\mathcal{A}(X)$ with $\left\|S_{\beta}^{*} R^{*}\right\| \leq$ $\left\|T^{*}\right\|\left\|R^{*}\right\|$ for all $\beta$ such that

$$
S_{\beta}^{*} \stackrel{\tau_{c}}{\longrightarrow} T^{*} .
$$

Hence by a standard argument (cf. [11, Lemma 3.3]) there exists a net $\left(T_{\alpha}\right)$ in $\operatorname{co}\left(\left\{S_{\beta}\right\}\right) \subset \mathcal{A}(X)$ such that

$$
T_{\alpha} \stackrel{\tau_{c}}{\longrightarrow} T \text { and } T_{\alpha}^{*} \stackrel{\tau_{c}}{\longrightarrow} T^{*},
$$

and it is also clear that $\left\|R T_{\alpha}\right\| \leq\|T\|\|R\|$ for all $\alpha$.

Proof of Theorem 2.4(a) $\Rightarrow$ (b). Let $Y$ be a finite-codimensional subspace of $X$. Let $\lambda>1$ be arbitrary. We show Proposition 2.2(c) to obtain the assertion that the pair $(X, Y)$ has the weak $\lambda$ - $\mathcal{A}$-BAP. Then in view of the definition of the weak BAP of pairs, the pair $(X, Y)$ also has the weak $\mathcal{A}$-MAP.

Now, let $Z$ be a Banach space and let $R \in \mathcal{W}(X, Z)$. Let $F$ be a finitedimensional subspace of $X$ and let $\varepsilon>0$. By (a) and Proposition 2.6, there exists a net $\left(T_{\alpha}\right)$ in $\mathcal{A}(X)$ with $\left\|R T_{\alpha}\right\| \leq\|R\|$ for all $\alpha$ such that $T_{\alpha} \stackrel{\tau_{c}}{\longrightarrow} i d_{X}$ and $T_{\alpha}^{*} \stackrel{\tau_{c}}{\longrightarrow}$ $i d_{X^{*}}$. We can find a finite rank projection $P: X \rightarrow X$ such that $P^{*}$ maps from $X^{*}$ onto the finite-dimensional space $Y^{\perp}:=\left\{x^{*} \in X^{*}: x^{*}(x)=0\right.$ for all $\left.x \in Y\right\}$. Let $\delta>0$ be such that $1+\delta\|P\|<\lambda$ and $\delta(1+\|P\|)<\varepsilon$. Since $T_{\alpha} \stackrel{\tau_{c}}{\longrightarrow} i d_{X}$ and $T_{\alpha}^{*} \stackrel{\tau_{c}}{\longrightarrow} i d_{X^{*}}$, there exists an $\alpha_{0}$ such that

$$
\left\|T_{\alpha_{0}} x-x\right\| \leq \delta \text { for all } x \in B_{F} \text { and }\left\|T_{\alpha_{0}}^{*} x^{*}-x^{*}\right\| \leq \delta \text { for all } x^{*} \in B_{Y^{\perp}} .
$$

Put $S=T_{\alpha_{0}}+P\left(i d_{X}-T_{\alpha_{0}}\right) \in \mathcal{A}(X)$. Then we have that

$$
\begin{aligned}
\|R S\| & =\left\|R T_{\alpha_{0}}+R P\left(i d_{X}-T_{\alpha_{0}}\right)\right\| \\
& \leq\|R\|+\|R\|\left\|P\left(i d_{X}-T_{\alpha_{0}}\right)\right\| \\
& =\|R\|+\|R\|\left\|\left(T_{\alpha_{0}}^{*}-i d_{X^{*}}\right) P^{*}\right\| \\
& \leq\|R\|(1+\delta\|P\|) \\
& \leq \lambda\|R\|,
\end{aligned}
$$


and for every $x \in F$

$$
\|S x-x\|=\left\|T_{\alpha_{0}} x-x+P\left(x-T_{\alpha_{0}} x\right)\right\| \leq \delta\|x\|+\delta\|P\|\|x\| \leq \varepsilon\|x\| .
$$

Moreover, since for all $x^{*} \in Y^{\perp}$

$$
S^{*} x^{*}=T_{\alpha_{0}}^{*} x^{*}+\left(i d_{X^{*}}-T_{\alpha_{0}}^{*}\right) P^{*} x^{*}=x^{*},
$$

we see that $S(Y) \subset Y$.

We need the following lemma to show Theorem $2.4(\mathrm{c}) \Rightarrow(\mathrm{a})$.

Lemma 2.8 ([10, Theorem 5.2]). Suppose that $\mathcal{A}(X)$ is a convex subset of $\mathcal{L}(X)$ and let $T \in \mathcal{L}(X)$. Then $T \in \overline{\mathcal{A}(X)}^{\tau_{c}}$ if and only if for every separable reflexive Banach space $Z$ and every $R \in \mathcal{K}(Z, X)$, we have $T R \in \overline{\{S R: S \in \mathcal{A}(X)\}}^{\tau_{c}}$.

Proof of Theorem $2.4(\mathrm{c}) \Rightarrow(\mathrm{a})$. First we assert that for every Banach space $Z$ and every $R \in \mathcal{W}(X, Z)$, for every finite-dimensional subspace $F$ of $X^{*}$ and for every $\varepsilon>0$, there exists an $S \in \mathcal{A}(X)$ with $\|R S\| \leq(\lambda+\varepsilon)\|R\|$ such that $S^{*} x^{*}=x^{*}$ for all $x^{*} \in F$.

In order to show the assertion, by (c), there exists a finite-codimensional subspace $Y$ of $X$ with $Y \subset F_{\perp}:=\left\{x \in X: x^{*}(x)=0\right.$ for all $\left.x^{*} \in F\right\}$ such that the pair $(X, Y)$ has the weak $\lambda$-A-BAP. Then $Y^{\perp}$ is finite-dimensional, $F \subset Y^{\perp}$, and we can choose a finite-dimensional subspace $E$ of $X$ such that $E^{\perp} \cap Y^{\perp}=\{0\}$.

Now there exists an $S \in \mathcal{A}(X)$ with $\|R S\| \leq(\lambda+\varepsilon)\|R\|$ such that $S x=x$ for all $x \in E$ and $S(Y) \subset Y$. Then a simple verification shows that $S^{*} x^{*}-x^{*} \in$ $E^{\perp} \cap Y^{\perp}=\{0\}$ for all $x^{*} \in Y^{\perp}$. Thus $S^{*} x^{*}=x^{*}$ for all $x^{*} \in F \subset Y^{\perp}$. This completes the assertion.

We now use Lemma 2.8 to show that $i d_{X^{*}} \in{\overline{\mathcal{A}^{*}(X)}}^{\tau_{c}}$. Let $Z$ be a reflexive Banach space and let $R \in \mathcal{K}\left(Z, X^{*}\right)$. Since $Z$ is reflexive, there exists a $U \in$ $\mathcal{K}\left(X, Z^{*}\right)$ such that $U^{*}=R$. Let $K$ be a compact subset of $Z$ and let $\varepsilon>0$. Choose a $\delta>0$ so that $(\delta+\delta(\lambda+\delta))\|U\| \leq \varepsilon$. Let $\left\{z_{i}\right\}_{i=1}^{n}$ be a $\delta$-net for $K$. Applying the above assertion to $U \in \mathcal{K}\left(X, Z^{*}\right)$ and $F=\operatorname{span}\left\{R z_{i}\right\}_{i=1}^{n}$, we obtain an $S \in \mathcal{A}(X)$ with $\|U S\| \leq(\lambda+\delta)\|U\|$ such that $S^{*} R z_{i}=R z_{i}$ for all $i=1, \ldots, n$. Then, for every $z \in K$, there exists a $z_{i_{0}}$ such that $\left\|z-z_{i_{0}}\right\| \leq \delta$. Thus we have that

$$
\begin{aligned}
\left\|R z-S^{*} R z\right\| & \leq\left\|R z-R z_{i_{0}}\right\|+\left\|R z_{i_{0}}-S^{*} R z\right\| \\
& \leq\|R\| \delta+\left\|S^{*} R z_{i_{0}}-S^{*} R z\right\| \\
& \leq\|R\| \delta+\delta\|U S\| \\
& \leq\|U\| \delta+\delta(\lambda+\delta)\|U\| \leq \varepsilon .
\end{aligned}
$$

Hence $R \in{\overline{\left\{S^{*} R: S \in \mathcal{A}^{*}(X)\right\}}}^{\tau_{c}}$.

The proof of [4, Proposition 1.6] actually shows the following more general result.

Theorem 2.9. Suppose that $\mathcal{A}(X)$ is a linear subspace of $\mathcal{L}(X)$, which contains $\mathcal{F}(X)$. Then the following statements are equivalent:

(a) $i d_{X^{*}} \in{\overline{\left\{S^{*} \in \mathcal{A}^{*}(X):\|S\| \leq \lambda\right\}}}^{\tau_{c}}$.

(b) For every finite-codimensional subspace $Y$ of $X$, the pair $(X, Y)$ has the $\lambda-\mathcal{A}-B A P$.

(c) For every finite-codimensional subspace $W$ of $X$, there exists a finite-codimensional subspace $Y$ of $X$ with $Y \subset W$ such that the pair $(X, Y)$ has the $\lambda-\mathcal{A}-B A P$. 


\section{A proof of Theorem 1.1}

We say that a Banach space $X$ has the weak $\lambda$ - $\mathcal{A}$-BAP if the space $\mathcal{F}(X)$ in the definition of the weak $\lambda$-BAP can be replaced by a linear subspace $\mathcal{A}(X)$ of $\mathcal{L}(X)$. In view of Proposition 2.2, if $\mathcal{A}(X)$ contains $\mathcal{F}(X)$, then $X$ has the weak $\lambda$-A-BAP if and only if for every Banach space $Z$ and every $R \in \mathcal{W}(X, Z)$, for every finite-dimensional subspace $F$ of $X$ and for every $\varepsilon>0$, there exists an $S \in \mathcal{A}(X)$ with $\|R S\| \leq(\lambda+\varepsilon)\|R\|$ such that $S x=x$ for all $x \in F$.

Proposition 3.1. Suppose that $\mathcal{A}=\mathcal{F}$ or $\mathcal{K}$. Let $Y$ be a finite-codimensional subspace of $X$. If $Y$ has the weak $\lambda-\mathcal{A}-B A P$, then the pair $(X, Y)$ has the weak $3 \lambda-\mathcal{A}-B A P$.

We need the following lemma, which is contained in the proof of [4, Proposition 1.8], to show Proposition 3.1.

Lemma 3.2. Let $Y$ be a finite-codimensional subspace of $X$ and let $\varepsilon>0$. Then there exists a finite-dimensional subspace $E$ of $X$ such that the map $Q: E \oplus_{1} Y \rightarrow X$ defined by $Q(e, y)=e+y$ is a $3(1+\varepsilon)$-quotient operator.

Proof of Proposition 3.1. Let $Z$ be a Banach space and let $R \in \mathcal{W}(X, Z)$. Let $F$ be a finite-dimensional subspace of $X$ and let $\varepsilon>0$. Choose a $\delta>0$ so that $(\lambda+\delta)(3+3 \delta)<3 \lambda+\varepsilon$.

Now, let $E$ and $Q$ be the objects in Lemma 3.2 for the $\delta>0$. We may assume that $F \supset E$ and $F \cap Y \neq\{0\}$. Since $Y$ has the weak $\lambda$-A-BAP, there exists a $T \in \mathcal{A}(Y)$ with $\left\|\left.R\right|_{Y} T\right\| \leq(\lambda+\delta)\|R\|$ such that $T y=y$ for all $y \in F \cap Y$. In view of the proof of [4, Proposition 1.8], the map $S: X \rightarrow X$ defined by $S x=e+T y$, where $Q(e, y)=x$, is well defined, $S x=x$ for all $x \in F$, and $S(Y) \subset Y$. Also it is easily seen that $S \in \mathcal{A}(X)$.

Now, let $x \in X$. Then by Lemma 3.2 there exist $e \in E$ and $y \in Y$ such that $Q(e, y)=x$ and $\|e\|+\|y\| \leq 3(1+\delta)\|x\|$. We now have that

$$
\begin{aligned}
\|R S x\| & =\|R e+R T y\| \\
& \leq\|R\|\|e\|+(\lambda+\delta)\|R\|\|y\| \\
& \leq(\lambda+\delta)\|R\|(\|e\|+\|y\|) \\
& \leq(\lambda+\delta)\|R\| 3(1+\delta)\|x\| \\
& \leq(3 \lambda+\varepsilon)\|R\|\|x\| .
\end{aligned}
$$

Hence the pair $(X, Y)$ has the weak $3 \lambda$ - $\mathcal{A}$-BAP.

Corollary 3.3. Suppose that $\mathcal{A}=\mathcal{F}$ or $\mathcal{K}$. If $i d_{X^{*}} \notin{\overline{\mathcal{A}^{*}(X)}}^{\tau_{c}}$, then for any $\lambda \geq 1$, there exists a finite-codimensional subspace $Y_{\lambda}$ of $X$ such that $Y_{\lambda}$ does not have the weak $\lambda-\mathcal{A}-B A P$.

Proof. Suppose that there exists a $\lambda \geq 1$ such that for every finite-codimensional subspace $Y$ of $X, Y$ has the weak $\lambda$-A-BAP, and so the pair $(X, Y)$ has the weak $3 \lambda$-A-BAP by Proposition 3.1. It follows from Theorem 2.4 that $i d_{X^{*}} \in \overline{\mathcal{A}}^{*}(X){ }^{\tau_{c}}$, which gives a contradiction.

Proof of Theorem 1.1. Since $X^{*}$ fails the AP, by Corollary 3.3, for each $n$, there exists a finite-codimensional subspace $Y_{n}$ of $X$ such that $Y_{n}$ fails to have the weak $n$-BAP. Since each $Y_{n}$ is complemented in $X$, it has the AP, and hence the desired 
space $Y=\left(\sum_{n} Y_{n}\right)_{c_{0}}\left(\operatorname{resp} .\left(\sum_{n} Y_{n}\right)_{\ell_{p}}(1 \leq p<\infty)\right)$ has the AP (cf. [1, Proposition 2.14]). But $Y$ fails to have the weak BAP.

A Banach space $X$ is said to have the compact approximation property (CAP) if $i d_{X} \in \overline{\mathcal{K}(X)}^{\tau_{c}}$. It is well known that the CAP is different from the AP (see [16]). The same proof of Theorem 1.1 yields the following:

Theorem 3.4. If $X$ has the $C A P$ but $i d_{X^{*}} \notin{\overline{\mathcal{K}^{*}(X)}}^{\tau_{c}}$, then there exists a subspace $Y$ of $c_{0}(X)$ (respectively, $\ell_{p}(X)(1 \leq p<\infty)$ ) such that $Y$ has the CAP but fails the weak $\mathcal{K}-B A P$.

\section{ACKNOWLEDGEMENT}

The authors would like to thank Professor W.B. Johnson and the referee for their helpful comments.

\section{REFERENCES}

[1] Peter G. Casazza, Approximation properties, Handbook of the geometry of Banach spaces, Vol. I, North-Holland, Amsterdam, 2001, pp. 271-316, DOI 10.1016/S1874-5849(01)80009-7. MR.1863695 (2003f:46012)

[2] Dong Yang Chen and Ben Tuo Zheng, Three-space problems for the bounded compact approximation property, Acta Math. Sin. (Engl. Ser.) 29 (2013), no. 4, 625-632, DOI 10.1007/s10114012-0661-7. MR.3029280

[3] T. Figiel and W. B. Johnson, The approximation property does not imply the bounded approximation property, Proc. Amer. Math. Soc. 41 (1973), 197-200. MR0341032 (49 \#5782)

[4] Tadeusz Figiel, William B. Johnson, and Aleksander Pełczyński, Some approximation properties of Banach spaces and Banach lattices, Israel J. Math. 183 (2011), 199-231, DOI 10.1007/s11856-011-0048-y. MR2811159

[5] Alexandre Grothendieck, Produits tensoriels topologiques et espaces nucléaires (French), Mem. Amer. Math. Soc. 1955 (1955), no. 16, 140. MR0075539 (17,763c)

[6] William B. Johnson, A complementary universal conjugate Banach space and its relation to the approximation problem, Proceedings of the International Symposium on Partial Differential Equations and the Geometry of Normed Linear Spaces (Jerusalem, 1972), Israel J. Math. 13 (1972), 301-310 (1973). MR0326356 (48 \#4700)

[7] William B. Johnson and Timur Oikhberg, Separable lifting property and extensions of local reflexivity, Illinois J. Math. 45 (2001), no. 1, 123-137. MR.1849989 (2002j:46014)

[8] W. B. Johnson, H. P. Rosenthal, and M. Zippin, On bases, finite dimensional decompositions and weaker structures in Banach spaces, Israel J. Math. 9 (1971), 488-506. MR0280983 (43 \#6702)

[9] Ju Myung Kim, A bounded approximation of weakly compact operators, J. Math. Anal. Appl. 401 (2013), no. 1, 154-159, DOI 10.1016/j.jmaa.2012.11.024. MR3011256

[10] Ju Myung Kim, The approximation properties via the Grothendieck p-compact sets, Math. Nachr. 286 (2013), no. 4, 360-373, DOI 10.1002/mana.201200054. MR.3028780

[11] Ju Myung Kim, The topology of pointwise, compact and weakly compact convergence on $\mathcal{B}(X, Y)$, Rocky Mountain J. Math. 40 (2010), no. 2, 543-559, DOI 10.1216/RMJ-2010-40-2543. MR2646457(2011c:47165)

[12] Åsvald Lima and Eve Oja, The weak metric approximation property, Math. Ann. 333 (2005), no. 3, 471-484, DOI 10.1007/s00208-005-0656-0. MR2198796 (2006i:46025)

[13] Joram Lindenstrauss and Lior Tzafriri, Classical Banach spaces. I, Springer-Verlag, Berlin, 1977. Sequence spaces; Ergebnisse der Mathematik und ihrer Grenzgebiete, Vol. 92. MR0500056 (58 \#17766)

[14] Eve Oja, The impact of the Radon-Nikodym property on the weak bounded approximation property (English, with English and Spanish summaries), RACSAM. Rev. R. Acad. Cienc. Exactas Fís. Nat. Ser. A Mat. 100 (2006), no. 1-2, 325-331. MR2267414 (2007h:46027) 
[15] A. Szankowski, Subspaces without the approximation property, Israel J. Math. 30 (1978), no. 1-2, 123-129, DOI 10.1007/BF02760833. MR508257 (80b:46032)

[16] George Willis, The compact approximation property does not imply the approximation property, Studia Math. 103 (1992), no. 1, 99-108. MR1184105 (93i:46035)

School of Mathematical Sciences, Xiamen University, Xiamen, 361005, People's RePUBLIC OF ChINA

E-mail address: cdy@xmu.edu.cn

Department of Mathematical Sciences, Seoul National University, Seoul, 151-747, KOREA

E-mail address: kjm21@kaist.ac.kr

Department of Mathematical Sciences, The University of Memphis, Memphis, TenNESSEE 38152-3240

E-mail address: bzheng@memphis.edu 